\title{
Impact of treatment of BPH on sexuality
}

\section{Schulman}

Prostate Cancer and Prostatic Diseases (2003) 6, 105. doi:10.1038/sj.pcan.4500633

Correction to: Prostate Cancer and Prostatic Diseases (2002), 4, Suppl 1, S12-S16. doi:10.1038/sj.pcan.

In the above paper the reference numbers that appeared in the legends for Figure 1, Figure 2, Figure 3, Figure 4 and Table 3 were incorrect.

The corrected legends appear below.

Figure 1 Effect of therapy on the ability to achieve an erection in the ALFIN Study. ${ }^{39,40}$
Figure 2 Effect of therapy on ejaculatory function in the ALFIN

Figure 3 Improvement in sexual score in patients with moderate symptoms of BPH after $1 y$ of treatment with alfuzosin. ${ }^{41}$

Figure 4 Improvement in sexual score in patients with severe symptoms after $1 y$ of treatment with alfuzosin. ${ }^{41}$

Table 3 Baseline characteristics of patients treated with alfuzosin $2.5 \mathrm{mg}$ t.i.d. or $5 \mathrm{mg}$ b.i.d. for $1 \mathrm{y}$ in the primary care setting ${ }^{41}$ 